\title{
Analysis of serum undercarboxylated osteocalcin level in rats with type 2 diabetes mellitus and the correlation with cognitive impairment
}

\author{
PENG-YING GU ${ }^{1}$, FENG YU ${ }^{2}$, SONG JIN ${ }^{1}$, QIAN YANG ${ }^{1}$, JING SU $^{1}$, \\ ${\text { YAN } \mathrm{CHEN}^{3} \text {, LIANG ZHAO }}^{4}$ and SHI-LIAN HU ${ }^{1}$ \\ Departments of ${ }^{1}$ Geriatric Medicine, ${ }^{2}$ Neurology, ${ }^{3}$ Endocrine Laboratory and ${ }^{4}$ Clinical Laboratory, \\ Anhui Provincial Hospital, Anhui Medical University, Hefei, Anhui 230001, P.R. China
}

Received March 31, 2017; Accepted July 13, 2017

DOI: $10.3892 /$ etm.2017.4838

\begin{abstract}
The aim of the study was to investigate the correlation between the serum undercarboxylated osteocalcin (ucOC) level and the blood biochemistry and cognitive impairment in rats with type 2 diabetes mellitus (T2DM). Sprague-Dawley (SD) rats were randomly divided into the normal control group (NC) and type 2 DM group. DM group received the high-fat and high-sugar diet combined with the intraperitoneal injection of low-dose STZ to establish the type 2 DM rat model. After 12 weeks of feeding, a Morris water maze was used to observe the rats' cognitive ability, and the levels of blood lipid, ucOC, insulin and adiponectin in the two groups were measured. The results showed that blood glucose of rats in DM group was increased significantly at $2-12$ weeks $(\mathrm{p}<0.01)$ and the body weight was significantly increased at $4-12$ weeks $(\mathrm{p}<0.01)$. The levels of serum triglyceride (TG), total cholesterol, low-density lipoprotein and insulin in rats in DM group were significantly increased compared with those in NC group $(\mathrm{p}<0.01)$ and the levels of high-density lipoprotein, adiponectin and ucOC were significantly decreased compared with those in the NC group $(\mathrm{p}<0.01)$. The place navigation and spatial exploration capacities of rats in DM group were significantly decreased compared with those in NC group $(\mathrm{p}<0.01)$. In the DM group, the place navigation and spatial exploration capacities of rats in the low ucOC group were significantly decreased compared with those in the high ucOC group $(\mathrm{p}<0.01)$. Additionally, single-factor correlation analysis revealed that ucOC was negatively correlated with blood glucose, TG and escape latency $(\mathrm{p}<0.01)$, but was positively correlated with adiponectin, residence time in target quadrant and traversing times
\end{abstract}

Correspondence to: Dr Shi-Lian Hu, Department of Geriatric Medicine, Anhui Provincial Hospital, Anhui Medical University, 17 Lujiang Road, Hefei, Anhui 230001, P.R. China

E-mail: kkhqxbmm2866052@163.com

Key words: type 2 diabetes mellitus, Sprague-Dawley rats, undercarboxylated osteocalcin, cognitive disorders $(p<0.05$ or $p<0.01)$. In conclusion, the decreased serum ucOC level in rats with type 2 diabetes mellitus has a certain correlation with cognitive impairment.

\section{Introduction}

Diabetes mellitus (DM) is a group of endocrine and metabolic diseases characterized by chronic blood sugar level increase due to the insufficiency of insulin secretion or insulin resistance in the body. With the improvement of living standards, an aging population and urbanization, have led to an increase of the morbidity of DM. Currently, there are approximately 366 million patients with type 2 DM (T2DM) worldwide. The International Diabetes Federation estimates that the number of DM patients is likely to reach 552 million patients by 2030 globally (1). The progression of DM, not only results in the acute and chronic complications of organs, such as eyes, nerves, kidneys, heart and blood vessels, eventually causing organ dysfunction and failure, but also have a certain influence on brain tissues, which is mainly manifested as changes in brain metabolism, structure and function $(2,3)$. In recent years, the correlation between DM and cognitive impairment has received attention from scholars in China and other countries. Previous findings have shown that the risk of cognitive impairment (Alzheimer's disease and vascular dementia) for DM patients is increased by 1.5 - to 2.0 -fold compared with healthy individuals, and its pathogenesis remains to be determined (4). Cognitive impairment often affects the life quality of DM patients and increases the difficulty of hypoglycemic therapy; thus, the study on pathogenic factors of DM cognitive impairment to identify effective treatment has far-reaching significance.

Undercarboxylated osteocalcin (ucOC) is a kind of non-collagen protein (4) that is mainly secreted by osteoblasts, affecting the body's glucolipid metabolism. Previous findings showed that the ucOC level is closely related to obesity, insulin resistance, diabetes and metabolic syndrome, and other endocrine and metabolic diseases (5). An animal study showed that the oral administration of exogenous ucOC for osteocalcin knockout mouse improved cognitive impairment, such as depression and anxiety (6). Considering that bone metabolism is affected by estrogen, this study established a male diabetic 
rat model to study the association between serum ucOC level and cognitive function and blood biochemistry in diabetic rats.

\section{Materials and methods}

Drugs. The following materials were used in the study: High-fat and high-sugar diet (including 58.5\% basal feed, 20\% sucrose, $18 \%$ lard, $2.5 \%$ cholesterol and $1 \%$ bile salt); blood glucose test strip (Roche, Mannheim, Germany); streptozotocin (STZ; Sigma-Aldrich, St. Louis, MO, USA); total cholesterol (TC), triglyceride (TG), high-density lipoprotein (HDL) and low-density lipoprotein (LDL) were all purchased from Nanjing Jiancheng Biological Engineering Institute (Nanjing, China); rat adiponectin ELISA kit (St. Charles, MO, USA); insulin ELISA kit (Beijing Atom Hi-Tech Co., Ltd., Beijing, China); and ucOC ELISA kit (Takara Bio Inc., Shiga, Japan).

Instrument. Instuments used in the study were: Ultramicroultraviolet spectrophotometer (Thermo Fisher Scientific, Inc., Waltham, MA, USA); continuous wavelength multi-functional microplate reader (TECAN, Salzburg, Austria); WMT-100 Morris water maze video analysis system (Chengdu Taimeng Science and Technology Co., Ltd., Chengdu, China); and low-temperature high-speed centrifugal machine (Thermo Fisher Scientific, Inc.).

Animals. Thirty male Sprague-Dawley (SD) rats of SPF level aged 4 weeks, were purchased from Shanghai Slack Experimental Animal Co., Ltd. (Shanghai, China) [license no. SCXK (Shanghai) 2012-0002]. The rats were fed independently on an SPF system and were housed at a temperature of $20 \pm 2^{\circ} \mathrm{C}$ and humidity of $50 \pm 5 \%$. The 12 -h circadian rhythm was controlled artificially. The animals had free access to food and water.

Animal modeling and grouping. After male SD rats were fed adaptively for 1 week, they were randomly divided into the normal control group $(\mathrm{NC}, \mathrm{n}=10)$ and diabetes mellitus group (DM, $\mathrm{n}=20)$ using random number table. The NC group was fed with normal diet and DM group was fed with high-fat and high-sugar diet for 6 weeks, followed by fasting for $12 \mathrm{~h}$. The DM group received an intraperitoneal injection of STZ ( $30 \mathrm{mg} / \mathrm{kg}$; prepared by $0.1 \mathrm{~mol} / \mathrm{l}$ and $\mathrm{pH} 4.2$ citric acid buffer) and the NC group received an intraperitoneal injection of equivalent citric acid buffer. After 3 days, blood was drawn from the caudal vein to measure the fasting blood glucose level. A diabetic model was established successfully when the blood glucose level was $>16.7 \mathrm{mmol} / \mathrm{l}$ (7). The rats were fed for another 6 weeks. The body weight and blood glucose level of the rats were monitored every 2 weeks.

Morris water maze test. At 12 weeks after modeling, the water maze test was conducted. The experiment was conducted for 5 consecutive days. A place navigation test was performed in the first 4 days, and a space exploration test was performed on the 5th day. The water maze was divided into four quadrants according to the ' + ' shape; and the platform was placed in the center of the 1st quadrant. The rats entered the water from the middle of the 2nd, 3rd and 4th quadrants and the escape latency was recorded, namely the time from searching the platform to climbing the platform. If no platform was found within $120 \mathrm{sec}$, the rats were placed on the platform for $20 \mathrm{sec}$ and then removed from the water maze, and the time was recorded as $120 \mathrm{sec}$. Each rat was trained once in the morning and afternoon, and the average was taken. On the 5th day, the platform in the 1st quadrant was removed, and the rats entered the water from the middle of the $2 \mathrm{nd}, 3 \mathrm{rd}$ and 4 th quadrants. The traversing times across the target quadrant (i.e., the 1st quadrant) within $120 \mathrm{sec}$ and the swimming time were recorded.

Detection of serum indexes. After the water maze test, the animals were fed with water but no food for $12 \mathrm{~h}$. Under anesthesia via the intraperitoneal injection of urethane, blood was drawn from the abdominal aorta, and the serum was separated and stored at $-80^{\circ} \mathrm{C}$ for subsequent use. TC, TG, HDL and LDL were measured according to kit protocol of the Nanjing Jiancheng Biological Engineering Institute. The continuous wavelength multifunctional microplate reader was used to determine the optical density (OD) value, the standard curve was drawn and the content was calculated. Serum was obtained to detect ucOC, insulin and adiponectin. The sample adding and treatment were conducted in strict accordance with the kit protocol. The OD value was measured at a wavelength of $450 \mathrm{~nm}$ using a continuous wavelength multifunctional microplate reader (Bio-Rad, Hercules, CA, USA), the standard curve was drawn, and the content of ucOC, insulin and adiponectin in each sample was calculated.

Statistical analysis. The experimental results were presented as mean \pm SD. SPSS 20.0 statistical software (Chicago, IL, USA) was used for statistical analysis of data. The independent sample t-test was used for intergroup comparison, and the Pearson or Spearman test was used for single-factor correlation analysis. $\mathrm{P}<0.05$ was considered to indicate a statistically significant difference.

\section{Results}

Comparison of blood glucose and body weight between the two groups. After the induction via high-sugar high-fat diet and STZ, the blood glucose levels of rats in DM group were significantly increased at 2-12 weeks compared with those in NC group, and the differences were statistically significant $(p<0.01)$. The body weight of rats in DM group was significantly increased compared with that in NC group, and the difference was statistically significant $(\mathrm{p}<0.01)$ (Figs. 1 and 2).

Comparison of levels of blood lipid, serum insulin, adiponectin and ucOC between the two groups. Compared with those in $\mathrm{NC}$ group, serum TG, TC, LDL and insulin levels of rats in the DM group were increased significantly, but HDL, adiponectin and ucOC levels were significantly decreased, and the differences were statistically significant $(\mathrm{p}<0.01)$ (Tables I and II).

Comparison of cognitive function between the two groups. In the water maze test, the escape latency of the DM group was significantly extended since the 2 nd day compared with that of NC group, and the difference was statistically significant $(p<0.01)$. In the space exploration test on the 5th day, the residence time in the target quadrant of the DM group was 


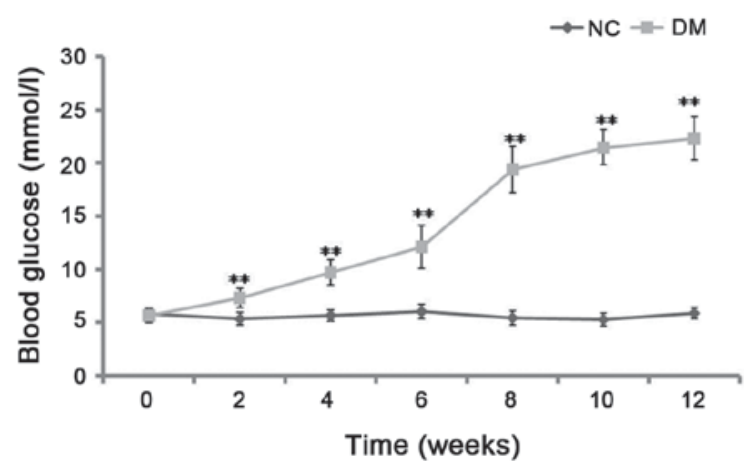

Figure 1. Comparison of blood glucose between the two groups. ${ }^{* *} \mathrm{P}<0.01$ compared with NC group. NC, normal control.

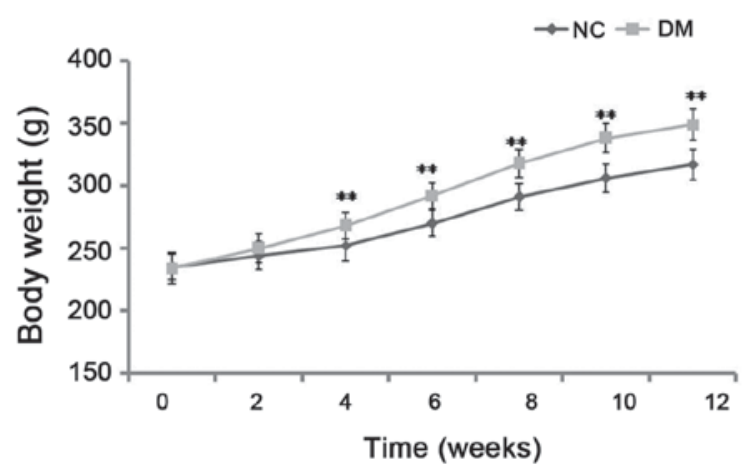

Figure 2. Comparison of body weight between the two groups. ${ }^{* *} \mathrm{P}<0.01$ compared with $\mathrm{NC}$ group. $\mathrm{NC}$, normal control.

Table I. Comparison of blood lipid levels between the two groups.

\begin{tabular}{lccccc}
\hline Group & Case & $\begin{array}{c}\mathrm{TC} \\
(\mathrm{mmol} / \mathrm{l})\end{array}$ & $\begin{array}{c}\mathrm{TG} \\
(\mathrm{mmol} / \mathrm{l})\end{array}$ & $\begin{array}{c}\mathrm{HDL} \\
(\mathrm{mmol} / \mathrm{l})\end{array}$ & $\begin{array}{c}\mathrm{LDL} \\
(\mathrm{mmol} / \mathrm{l})\end{array}$ \\
\hline $\mathrm{NC}$ & 10 & $2.58 \pm 0.23$ & $0.87 \pm 0.09$ & $1.31 \pm 0.11$ & $1.06 \pm 0.08$ \\
$\mathrm{DM}$ & 20 & $5.59 \pm 0.41^{\mathrm{a}}$ & $1.75 \pm 0.14^{\mathrm{a}}$ & $0.72 \pm 0.08^{\mathrm{a}}$ & $1.90 \pm 0.17^{\mathrm{a}}$
\end{tabular}

${ }^{a} \mathrm{P}<0.01$ compared with NC group. TC, total cholesterol; TG, triglyceride; HDL, high-density lipoprotein; LDL, low-density lipoprotein; NC, normal control; DM, diabetes mellitus.

significantly shortened compared with that of the NC group, and the traversing times across the target quadrant were significantly decreased; the differences were statistically significant $(\mathrm{p}<0.01)$ (Tables III and IV).

Comparison of cognitive function between the low ucOC and high ucOC groups of DM rats. According to the mean ucOC, DM group was divided into the low ucOC and high ucOC groups, and the cognitive function of rats between the two groups was compared. In the place navigation test, the escape latencies of the low ucOC group on the 3rd and 4th day were significantly prolonged compared with those of the high ucOC group, and the differences were statistically significant $(\mathrm{p}<0.01)$. In the space exploration test on the
Table II. Comparison of serum insulin, adiponectin and ucOC levels between the two groups.

\begin{tabular}{lcccc}
\hline Group & Case & $\begin{array}{c}\text { Insulin } \\
(\mu \mathrm{IU} / \mathrm{ml})\end{array}$ & $\begin{array}{c}\text { Adiponectin } \\
(\mu \mathrm{g} / \mathrm{ml})\end{array}$ & $\begin{array}{c}\text { ucOC } \\
(\mathrm{pg} / \mathrm{ml})\end{array}$ \\
\hline $\mathrm{NC}$ & 10 & $43.09 \pm 8.38$ & $1.90 \pm 0.27$ & $59.82 \pm 6.61$ \\
$\mathrm{DM}$ & 20 & $107.34 \pm 11.16^{\mathrm{a}}$ & $1.05 \pm 0.22^{\mathrm{a}}$ & $44.57 \pm 5.30^{\mathrm{a}}$ \\
\hline
\end{tabular}

${ }^{\text {a }}<0.01$ compared with NC group. ucOC, undercarboxylated osteocalcin.

Table III. Comparison of escape latency between the two groups.

\begin{tabular}{llcccc}
\hline & \multicolumn{4}{c}{ Escape latency (sec) } \\
\cline { 3 - 6 } Group & Case & 1st day & 2nd day & 3rd day & 4th day \\
\hline NC & 10 & $75.97 \pm 12.21$ & $56.26 \pm 10.65$ & $37.02 \pm 8.66$ & $25.53 \pm 7.01$ \\
DM & 20 & $88.13 \pm 10.16$ & $85.49 \pm 9.33^{\mathrm{a}}$ & $62.60 \pm 10.94^{\mathrm{a}}$ & $56.39 \pm 9.19^{\mathrm{a}}$
\end{tabular}

${ }^{\mathrm{a}} \mathrm{P}<0.01$ compared with $\mathrm{NC}$ group. $\mathrm{NC}$, normal control; DM, diabetes mellitus.

Table IV. Comparison of space exploration between the two groups.

\begin{tabular}{lccc}
\hline Group & Case & $\begin{array}{c}\text { Residence time in } \\
\text { target quadrant }(\%)\end{array}$ & $\begin{array}{c}\text { Traversing } \\
\text { times }\end{array}$ \\
\hline $\mathrm{NC}$ & 10 & $33.78 \pm 6.05$ & $9.35 \pm 2.14$ \\
$\mathrm{DM}$ & 20 & $17.29 \pm 6.82^{\mathrm{a}}$ & $2.47 \pm 0.83^{\mathrm{a}}$ \\
\hline
\end{tabular}

${ }^{\mathrm{a}} \mathrm{P}<0.01$ compared with $\mathrm{NC}$ group. $\mathrm{NC}$, normal control; DM, diabetes mellitus.

5th day, the residence time in the target quadrant of the low ucOC group was significantly shortened compared with that of the high ucOC group, and the traversing times across the target quadrant were significantly decreased; the differences were statistically significant $(\mathrm{p}<0.01)$ (Tables V and VI).

Analysis of correlation between ucOC of the DM group and blood biochemistry, insulin, adiponectin and cognitive function. Single-factor correlation analysis revealed that ucOC was negatively correlated with blood glucose, TG and escape latency $(p<0.01)$, but was positively correlated with adiponectin, residence time in target quadrant and traversing times $(\mathrm{p}<0.05$ or $\mathrm{p}<0.01)$ (Table VII).

\section{Discussion}

DM is a kind of endocrine and metabolic disease, and T2DM patients account for more than $90 \%$ of DM patients in China (3). The pathogenesis of T2DM mainly includes the insufficiency of insulin secretion and insulin resistance. The adiponectin is secreted by adipocytes, its expression level in 
Table V. Comparison of escape latency between the low ucOC and high ucOC groups.

\begin{tabular}{lccccc}
\hline & \multicolumn{4}{c}{ Escape latency (sec) } \\
\cline { 2 - 6 } Group Case & 1st day & 2nd day & 3rd day & 4th day \\
\hline Low & 10 & $92.64 \pm 11.27$ & $89.62 \pm 8.97$ & $70.43 \pm 12.98$ & $64.75 \pm 9.03$ \\
ucOC & & & & & \\
High & 10 & $83.62 \pm 9.05$ & $81.36 \pm 9.69$ & $54.77 \pm 8.90^{\text {a }}$ & $48.03 \pm 9.35$ \\
ucOC & & & & & \\
\hline
\end{tabular}

${ }^{\mathrm{a}} \mathrm{P}<0.01$ compared with low ucOC group. ucOC, undercarboxylated osteocalcin.

Table VI. Comparison of space exploration between low ucOC group and high ucOC group.

\begin{tabular}{lccc}
\hline Group & Case & $\begin{array}{c}\text { Residence time in } \\
\text { target quadrant }(\%)\end{array}$ & $\begin{array}{c}\text { Traversing } \\
\text { times }\end{array}$ \\
\hline Low ucOC & 10 & $13.47 \pm 6.35$ & $1.60 \pm 0.85$ \\
High ucOC & 10 & $21.11 \pm 7.29^{\mathrm{a}}$ & $3.34 \pm 0.81^{\mathrm{a}}$ \\
\hline
\end{tabular}

${ }^{\mathrm{a}} \mathrm{P}<0.01$ compared with low ucOC group. ucOC, undercarboxylated osteocalcin.

Table VII. Analysis of the correlation between ucOC and blood biochemistry, insulin, adiponectin and cognitive function.

\begin{tabular}{lrr}
\hline Index & r value & P-value \\
\hline Blood sugar & -0.476 & 0.002 \\
TG & -0.602 & 0.000 \\
Adiponectin & 0.296 & 0.017 \\
Escape latency (3rd day) & -0.518 & 0.001 \\
Escape latency (4th day) & -0.577 & 0.001 \\
Residence time in & 0.610 & 0.000 \\
target quadrant & & \\
Traversing times & 0.489 & 0.001 \\
\hline
\end{tabular}

ucOC, undercarboxylated osteocalcin; TG, triglyceride.

the body is negatively correlated with body fat content and it has an insulin-like metabolic effect. In the body, adiponectin plays a role of reducing blood glucose, lipid and blood pressure, anti-inflammation, improving insulin resistance and anti-atherosclerosis. At present, there are mainly two kinds of T2DM animal models in China and other countries. The first type of animal model is the DM gene knockout animals or inbred animals with genetic predisposition, such as ob/ob mice, $\mathrm{db} / \mathrm{db}$ mice, Zucker rats and OLETF rats. The second type of animal model is induced by the injection of small-dose STZ numerous times $(20-35 \mathrm{mg} / \mathrm{kg})$ to destroy the islet cells or the short-term high-fat and high-sugar diet combined with injection of high-dose STZ ( $>50 \mathrm{mg} / \mathrm{kg}$ ) (8). In the present study, SD rats were fed a high-fat and high-sugar diet for 6 weeks to induce hyperinsulinemia and insulin resistance, followed by intraperitoneal injection of small-dose STZ to induce the hyperglycemic state eventually. After 12 weeks of induction, weight gain, hyperglycemia, hyperinsulinemia, dyslipidemia and decreased adiponectin appeared in rats in DM group. Thus the clinical manifestations and blood biochemical levels of most clinical T2DM patients were successfully reproduced.

The progression of disease can cause retinopathy, nephropathy, heart disease, peripheral vascular lesions and nervous lesion, as well as other common chronic complications (2). In recent years, it has been shown that T2DM patients may suffer from different degrees of cognitive impairment, manifested as inattention, decreased learning and memory, reaction capacity and mental activity $(9,10)$. Another study has found that DM patients may suffer from the impaired cerebral frontal lobe function, including the decreased mental activity, executive capacity and processing speed (11). The risk of dementia for T2DM patients increases by 1-fold compared with healthy individuals (12). By contast, T2DM can accelerate the progression of patients with cognitive impairment and develop into dementia rapidly (13). This animal experiment found that in the place navigation test, the latent time of rats in DM group was significantly prolonged, and in the space exploration test on the 5th day, the residence time in the target quadrant of rats in DM group was significantly shortened and the traversing times across the target quadrant were significantly decreased, indicating that DM rats suffer from the cognitive impairment.

Osteocalcin (OC), also known as bone glutamate protein, is a kind of non-collagen protein, composed of 49 amino acids (14). Its three glutamic acid residues in amino acid sites can be carboxylated under the catalysis of $\gamma$-carboxylase. If all three glutamic acid residues are carboxylated, completely carboxylated OC occurs, and fewer than three glutamic acid residues of ucOC are carboxylated (15). The present study has found that ucOC is involved in the body's glycolipid metabolism (16). Additionally, the serum OC level was negatively correlated with obesity, insulin resistance, diabetes and metabolic syndrome (5). Ferron and Lacombe studied the correlation between serum ucOC level and metabolic indexes, and found that the serum ucOC level was positively correlated with adiponectin, HDL and insulin level, but negatively correlated with blood glucose, body mass index, glycosylated hemoglobin, TC, TG and inflammatory indexes (5). Another human trial has also found that T2DM patients may suffer from decreased serum OC level (17). In animal studies, it was found that OC-knockout mice suffered from decreased blood glucose, impaired glucose tolerance, decreased insulin secretion and sensitivity and lipid metabolism disorder and after exogenous ucOC was administered, the above situation was improved (16). The improvement was likely due to the high glucose toxicity inhibiting osteoblast activity (18), leading to the reduction of OC synthesis and secretion. This animal experiment also found that the serum ucOC level of rats in DM group was significantly decreased compared with that in NC group and serum ucOC level was negatively correlated with blood glucose and TG, but positively correlated with adiponectin, which was consistent with the clinical studies of previous scholars. 
Runx 2 is the main gene regulating OC expression (19). Aat the same time, the Runx2 gene mutation can cause cleidocranial dysplasia (20). In clinical studies, it was found that patients with cleidocranial dysplasia may suffer from cognitive impairments (21). Animal experiments have shown that OC-knockout mice may suffer from decreased memory and spatial learning capacities as well as an anxious and depressed state. When exogenous ucOC is administered, the anxious and depressed behaviors and learning and memory deficits are improved. It is considered that $\mathrm{OC}$ is able to permeate the blood-brain barrier and bind to neurons in the midbrain, hippocampus and brainstem, to promote the synthesis and secretion of neurotransmitters and inhibit the synthesis of $\gamma$-aminobutyric acid, thus producing anti-anxiety and anti-depression effects, and promoting the improvement of learning and memory capacities. Puig et al (22) studied obese women and found that the serum OC level in obese women is significantly decreased compared with that in healthy women. Additionally, using an MRI and neuropsychological scale, those authors found that low-level OC can cause changes in brain microstructure and decrease in cognitive function. This animal experiment showed that the serum ucOC level in DM rats was negatively correlated with the escape latency, but positively correlated with the residence time in target quadrant and traversing times, indicating that $\mathrm{ucOC}$ has a protective effect on cognitive function of DM rats.

In conclusion, DM rats may suffer from a glucose and lipid metabolism disorder, decreased serum adiponectin, ucOC level and cognitive function. Serum ucOC level is negatively correlated with blood glucose and TG, but positively correlated with adiponectin. At the same time, decreased serum ucOC has a certain correlation with cognitive impairment. The present study was conducted only from the correlation between serum level and cognitive function; thus, the mechanism affecting the cognitive function remains to be further studied.

\section{Acknowledgements}

The present study was supported by Anhui Provincial Health Department (ID, 13zc010).

\section{References}

1. Shorr RI, de Rekeneire N, Resnick HE, Yaffe K, Somes GW, Kanaya AM, Simonsick EM, Newman AB and Harris TB: Glycemia and cognitive function in older adults using glucoselowering drugs. J Nutr Health Aging 10: 297-301, 2006.

2. Manschot SM, Biessels GJ, Rutten GE, Kessels RP, Gispen WH and Kappelle LJ; Utrecht Diabetic Encephalopathy Study Group: Peripheral and central neurologic complications in type 2 diabetes mellitus: No association in individual patients. J Neurol Sci 264: 157-162, 2008.

3. Verdile G, Fuller SJ and Martins RN: The role of type 2 diabetes in neurodegeneration. Neurobiol Dis 84: 22-38, 2015.
4. Arvanitakis Z, Wilson RS, Bienias JL, Evans DA and Bennett DA: Diabetes mellitus and risk of Alzheimer disease and decline in cognitive function. Arch Neurol 61: 661-666, 2004.

5. Ferron M and Lacombe J: Regulation of energy metabolism by the skeleton: Osteocalcin and beyond. Arch Biochem Biophys 561: 137-146, 2014.

6. Oury F, Khrimian L, Denny CA, Gardin A, Chamouni A, Goeden N, Huang YY, Lee H, Srinivas P, Gao XB, et al: Maternal and offspring pools of osteocalcin influence brain development and functions. Cell 155: 228-241, 2013.

7. Elias D, Prigozin H, Polak N, Rapoport M, Lohse AW and Cohen IR: Autoimmune diabetes induced by the beta-cell toxin STZ. Immunity to the $60-\mathrm{kDa}$ heat shock protein and to insulin. Diabetes 43: 992-998, 1994.

8. Park SH, Marso SP, Zhou Z, Foroudi F, Topol EJ and Lincoff AM: Neointimal hyperplasia after arterial injury is increased in a rat model of non-insulin-dependent diabetes mellitus. Circulation 104: 815-819, 2001.

9. Convit A: Links between cognitive impairment in insulin resistance: An explanatory model. Neurobiol Aging 26: 31-35, 2005

10. Kodl CT and Seaquist ER: Cognitive dysfunction and diabetes mellitus. Endocr Rev 29: 494-511, 2008.

11. Christman AL,Matsushita K, Gottesman RF,Mosley T, Alonso A, Coresh J, Hill-Briggs F, Sharrett AR and Selvin E: Glycated haemoglobin and cognitive decline: The Atherosclerosis Risk in Communities (ARIC) study. Diabetologia 54: 1645-1652, 2011.

12. Biessels GJ, Staekenborg S, Brunner E, Brayne C and Scheltens P: Risk of dementia in diabetes mellitus: A systematic review. Lancet Neurol 5: 64-74, 2006.

13. Alagiakrishnan K and Sclater A: Psychiatric disorders presenting in the elderly with type 2 diabetes mellitus. Am J Geriatr Psychiatry 20: 645-652, 2012.

14. Hauschka PV, Lian JB, Cole DE and Gundberg CM: Osteocalcin and matrix Gla protein: Vitamin K-dependent proteins in bone. Physiol Rev 69: 990-1047, 1989.

15. Patti A, Gennari L, Merlotti D, Dotta F and Nuti R: Endocrine actions of osteocalcin. Int J Endocrinol 2013: 846480, 2013.

16. Lee NK, Sowa H, Hinoi E, Ferron M, Ahn JD, Confavreux C, Dacquin R, Mee PJ, McKee MD, Jung DY, et al: Endocrine regulation of energy metabolism by the skeleton. Cell 130: 456-469, 2007.

17. Villafán-Bernal JR, Llamas-Covarrubias MA, Muñoz-Valle JF, Rivera-León EA, González-Hita ME, Bastidas-Ramírez BE, Gurrola-Díaz CM, Armendáriz-Borunda JS and SánchezEnríquez S: A cut-point value of uncarboxylated to carboxylated index is associated with glycemic status markers in type 2 diabetes. J Investig Med 62: 33-36, 2014.

18. Botolin S and McCabe LR: Chronic hyperglycemia modulates osteoblast gene expression through osmotic and non-osmotic pathways. J Cell Biochem 99: 411-424, 2006.

19. Okazaki K and Sandell LJ: Extracellular matrix gene regulation. Clin Orthop Relat Res 427: S123-S128, 2004.

20. Yoshida T, Kanegane H, Osato M, Yanagida M, Miyawaki T, Ito Y and Shigesada K: Functional analysis of RUNX2 mutations in Japanese patients with cleidocranial dysplasia demonstrates novel genotype-phenotype correlations. Am J Hum Genet 71: 724-738, 2002 .

21. McBrien H, Turk $J$ and Letch N: The management of ADHD and associated problems in a young person with cleidocranial dysostosis (CCD) and mild intellectual disability. Clin Child Psychol Psychiatry 11: 445-456, 2006.

22. Puig J, Blasco G, Daunis-i-Estadella J, Moreno M, Molina X, Alberich-Bayarri A, Xifra G, Pedraza S, Ricart W, FernándezAranda $\mathrm{F}$, et al: Lower serum osteocalcin concentrations are associated with brain microstructural changes and worse cognitive performance. Clin Endocrinol (Oxf) 84: 756-763, 2016. 